\title{
The utility of three-dimensional computed tomography for prediction of tumor invasiveness in clinical stage IA lung adenocarcinoma
}

\author{
Yohei Kawaguchi, Masayuki Nakao, Kenshiro Omura, Naoya Iwamoto, Hiroki Ozawa, Yasuto Kondo, \\ Junji Ichinose, Yosuke Matsuura, Sakae Okumura, Mingyon Mun
}

Department of Thoracic Surgical Oncology, Cancer Institute Hospital, The Japanese Foundation for Cancer Research, Tokyo, Japan

Contributions: (I) Conception and design: Y Kawaguchi; (II) Administrative support: M Nakao; (III) Provision of study materials or patients: Y Kawaguchi; (IV) Collection and assembly of data: Y Kawaguchi; (V) Data analysis and interpretation: Y Kawaguchi, M Nakao; (VI) Manuscript writing: All authors; (VII) Final approval of manuscript: All authors.

Correspondence to: Masayuki Nakao, MD. Department of Thoracic Surgical Oncology, Cancer Institute Hospital, The Japanese Foundation for Cancer Research, Tokyo, Japan. Email: masayuki.nakao@jfcr.or.jp.

\begin{abstract}
Background: It is critical to have an accurate measurement of solid tumor size in order to predict the invasiveness of small lung adenocarcinomas. Some lesions cannot be measured accurately via Highresolution computed tomography (HRCT) due to their irregular shape and unclear borders. For this reason, we evaluated the relative efficacy of three-dimensional (3D) CT for predicting invasive adenocarcinoma.

Methods: We evaluated 195 patients with clinical stage IA adenocarcinomas, including 109 with lesions documented as invasive that were surgically resected at our institute during 2017. All lesions were categorized as either (I) lesions that were difficult to evaluate (i.e., hazy lesions; HL) or (II) more typical lesions (TL). The relationships between solid tumor size as determined by HRCT, solid tumor volume as determined by 3D CT and pathologic diagnosis were evaluated.

Results: Fifty-seven patients (29\%) were diagnosed with HL. We set the cut-off value for the solid volume at $225 \mathrm{~mm}^{3}$ as predictive for invasive adenocarcinoma. When evaluating all 195 patients as a group, the accuracy, sensitivity, and specificity based on the solid tumor volume were similar to those based on the solid tumor size. When we limit our analysis to the HL group, the specificity based on solid tumor volume (65.5\%) was higher than that based on solid tumor size $(44.8 \%)$ with a difference that approached statistical significance $(\mathrm{P}=0.070)$.
\end{abstract}

Conclusions: 3D CT was equivalent to HRCT for predicting invasive adenocarcinoma and may be particularly useful for diagnosing lesions that are difficult to evaluate on HRCT.

Keywords: Three-dimensional computed tomography (3D CT); invasive adenocarcinoma; high-resolution computed tomography (HRCT); radiology; Synapse Vincent

Submitted Jun 05, 2020. Accepted for publication Oct 10, 2020.

doi: $10.21037 /$ jtd-20-2131

View this article at: http://dx.doi.org/10.21037/jtd-20-2131

\section{Introduction}

With recent advances in diagnostic imaging, accumulating evidence suggests that preoperative high-resolution computed tomography (HRCT) will be extremely useful for the evaluation of pathological malignant grade and prognostic outcomes in patients with peripherally-located small lung adenocarcinomas (1-5). These lesions are frequently visualized radiologically as part-solid nodules on HRCT. HRCT measurements that focus on the solid part of the nodule (i.e., solid tumor size) have been reported to predict pathologic invasiveness and are better prognostic indicators than measurements of whole tumor size 
$(1-4,6,7)$. As such, this measurement has been introduced as a component of the $\mathrm{T}$ factor determination in the 8th Edition of the TNM Classification for Lung and Pleural Tumors of the Union for International Cancer Control (TNM 8th) (8). Histologically, adenocarcinoma is classified into preinvasive lesions, such as adenocarcinoma in situ (AIS), minimally invasive adenocarcinoma (MIA), and invasive adenocarcinoma largely based on size and invasiveness (9-11). Patients with either AIS or MIA typically experience no recurrences if complete resection is achieved; these patients may be good candidates for intentional limited resection $(9,12)$. As such, it is important to be able to distinguish borders of the solid tumor for specific and precise measurement; this information is critical for appropriate surgical management.

In our clinical practices, we often encounter lesions that are difficult to evaluate on HRCT because of their irregular shape and unclear borders of the solid and non-solid parts. These lesions frequently subject to intra- and interobserver variability when determining the clinical $\mathrm{T}$ factor. To address this problem, we have introduced preoperative three-dimensional (3D) CT imaging technology. 3D CTs are becoming increasingly common in daily practice and can promote both safe and accurate surgical procedures as well as provide important operative simulations and navigation (13-17). Given the aforementioned diagnostic problems with HRCT, this technology may also be useful for documenting accurate sized of small lung adenocarcinomas and thereby predicting pathological outcomes. 3D CT is a semi-automatic modality (Fujifilm Synapse Vincent system; Fujifilm Corporation, Tokyo, Japan) that readily facilitates quantification of the both solid and non-solid volumes of small neoplastic lesions in the lung and thus may be superior to HRCT for this indication $(13,14,18,19)$.

Here, we compared the efficacies of HRCT and 3D CT imaging for predicting invasive lung adenocarcinoma, with special attention to the lesions that are difficult to evaluate using the former modality. We present the following article in accordance with the STROBE reporting checklist (available at http://dx.doi.org/10.21037/jtd-20-2131).

\section{Methods}

\section{Patients}

We identified 207 patients with clinical stage IA (TNM 8 th) adenocarcinomas who underwent surgical resection at our institute from January through December of 2017.
We excluded 12 patients who did not undergo HRCT at our hospital. The remaining 195 patients (with 195 lesions) were included in this study. The study was conducted in accordance with the Declaration of Helsinki (as revised in 2013). Data collection and analysis were approved by the Institutional Review Board of the Cancer Institute Hospital of Japanese Foundation for Cancer Research (2019-1235). The need to obtain written informed consent from each patient was waived because of the retrospective nature of the study and anonymity of the subjects.

\section{Pathologic classification}

All of surgical specimens were fixed in $20 \%$ formalin and embedded in paraffin. Representative sections were routinely stained with hematoxylin and eosin (H\&E). Experienced pathologists reviewed the samples to confirm that the sections contained carcinoma cells, and measured longest overall diameter as well as the invasive component diameter and classified the lesions as AIS, MIA, or invasive adenocarcinoma.

\section{HRCT assessment}

All patients and lesions were evaluated by HRCT. The most frequently used unit was the 64-section multidetector CT scanner (Discovery CT750HD; GE Healthcare, Milwaukee, WI, USA). Images were reconstructed with a section thickness of $1.25 \mathrm{~mm}$ in lung window settings [level $=-500$ Hounsfield units $(\mathrm{HU})$; width $=1,500 \mathrm{HU}]$. We measured the longest diameters of the solid and nonsolid components. The non-solid component, typically ground glass opacity, was defined as a hazy increase in lung attenuation that did not obscure the underlying vascular markings. We categorized the 195 lesions into two groups. One group includes the lesions which were difficult to evaluate due to irregular and hazy shapes, with unclear boundaries between solid and non-solid regions (hazy lesion; HL). Those remaining were the more typical lesions which were comparatively easy to evaluate because the boundaries between solid and non-solid regions were clear (typical lesion: TL; Figure 1). Measurements of the solid regions were performed by two experienced thoracic surgeons (Y Kawaguchi, M Nakao). If there was a discrepancy between the observer results, the two discussed the case and made a final determination. The assignment of each nodule to either the HL or TL group was also made by consensus. 

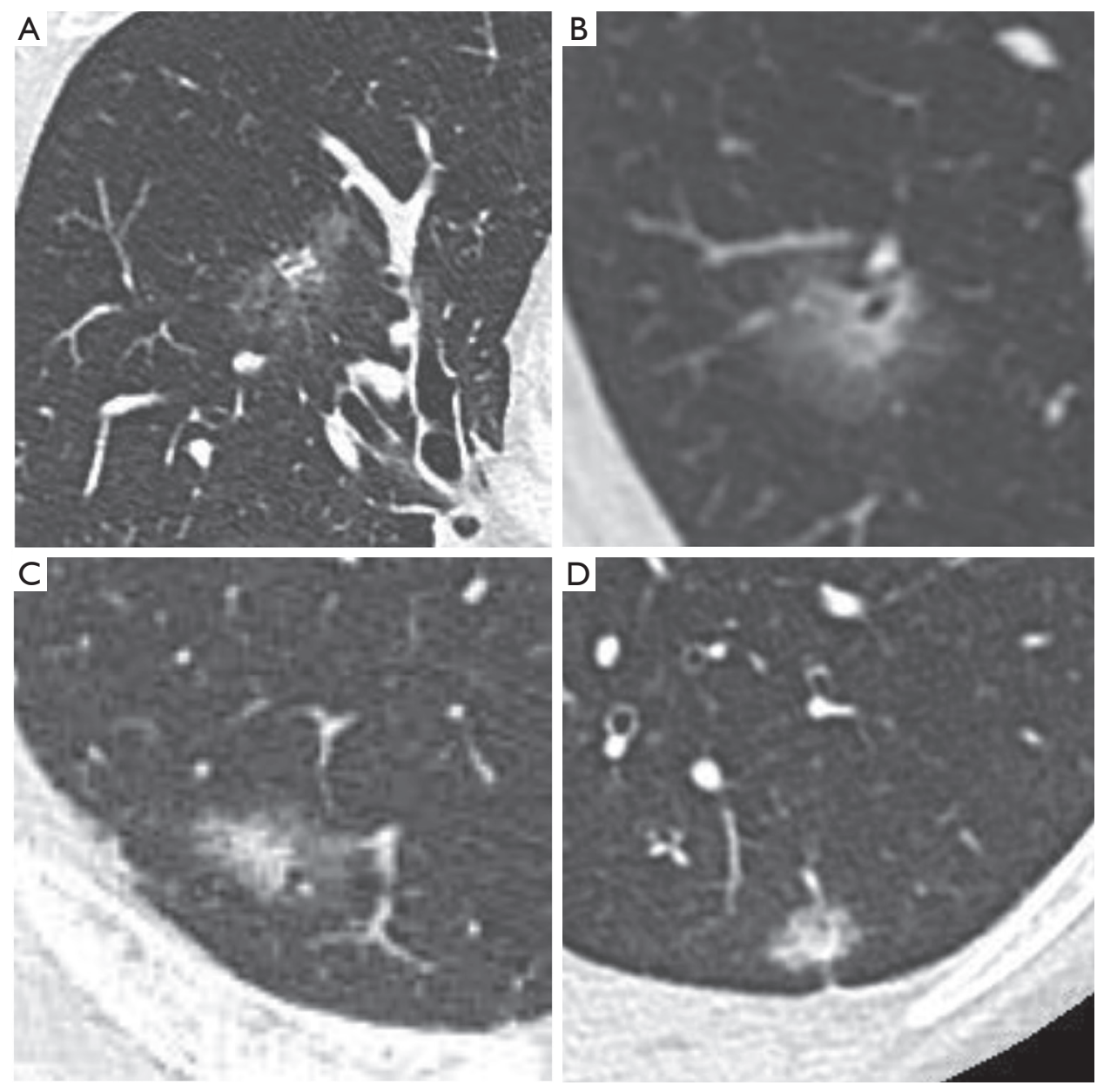

Figure 1 Categorizing images on HRCT. (A,B) Lesions in which the solid region could be readily distinguished from the non-solid regions were defined as TL. (C,D) Lesions in which the boundaries between the solid regions and the non-solid regions were unclear and/or the distinction between the solid regions and the non-solid regions were difficult to define were categorized as hazy lesions (HL). HRCT, highresolution computed tomography; TL, typical lesions.

\section{D CT assessment by the Synapse Vincent}

CT imaging data were transferred to a workstation with the Synapse Vincent software. The 3D images were constructed and $3 \mathrm{D}$ volumetric analysis was performed semi-automatically. $3 \mathrm{D}$ constructed tumor imaging provided us with an assessment of the whole tumor volume, the solid tumor volume and the non-solid volume based on density levels (Figure 2). For volumetric analysis of a lung nodule, a density level of $-300 \mathrm{HU}$ is the threshold level that separated solid and non-solid regions by the software algorithm. The relationships between the size of the solid tumor region on HRCT, the solid tumor volume on $3 \mathrm{D}$ CT, and pathological diagnosis (AIS, MIA or invasive adenocarcinoma) were analyzed.

\section{Statistical analysis}

The receiver operating characteristic (ROC) curve of solid volume was used to predict invasiveness. The area under the ROC curve (AUC) and its $95 \%$ confidence interval (CI) were measured. The cut-off value to predict adenocarcinoma invasiveness was defined as $5 \mathrm{~mm}$ according to the TNM 8th. Inter-observer variability was included in the calculation of interclass correlation coefficient (ICC). Accuracy rate, sensitivity, and specificity for the prediction of invasive adenocarcinoma were calculated based on the size and volume of the solid region. The McNemar test 

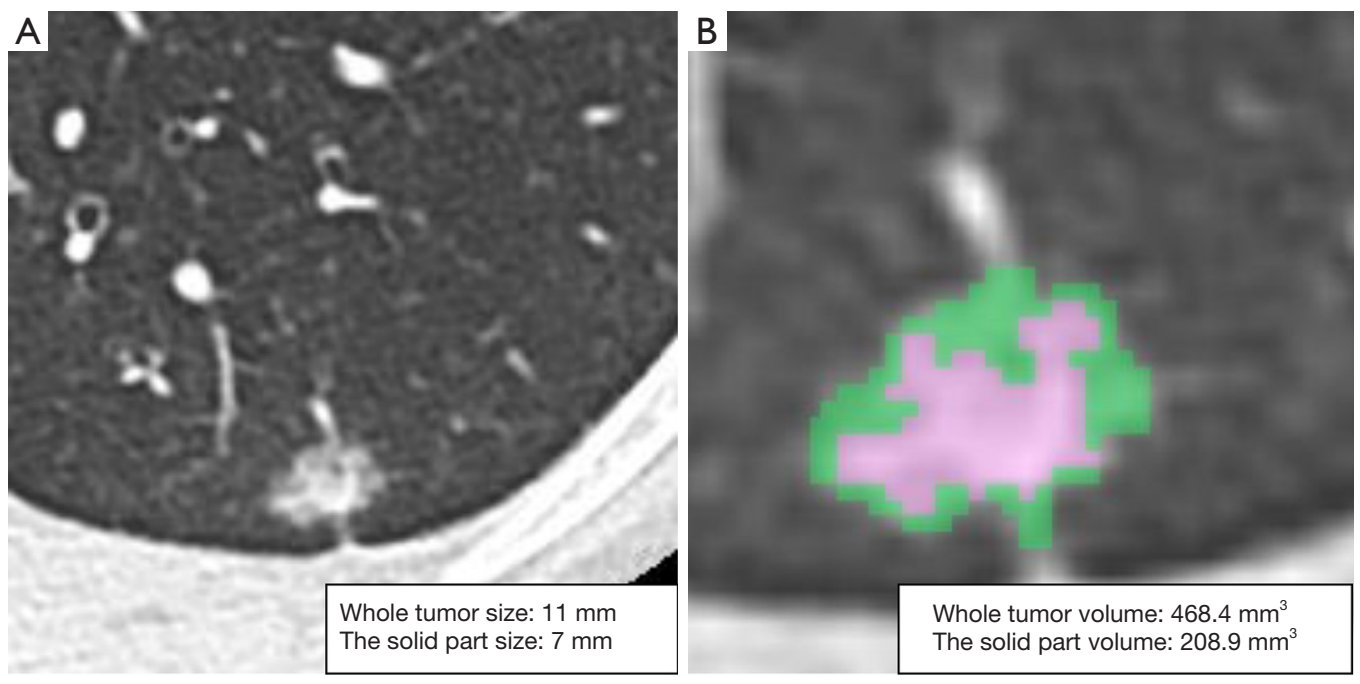

Figure 2 Representative images of the quantification of the lung tumor volume with 3D imaging analysis using the Synapse Vincent system. (A) Two dimensional; (B) the 3D imaging results.

was used to compare accuracy, sensitivity, and specificity between each method; $\mathrm{P}$ values of $<0.05$ were considered to indicate statistical significance. SPSS statistical software package (version 24.0; DDR3 RDIMM, SPSS Inc., Chicago, IL, USA) was used for statistical analysis.

\section{Results}

The characteristics of all patients investigated in this study are shown in Table 1. Eighty-six patients (44\%) were diagnosed with AIS or MIA and 109 patients (56\%) were diagnosed with invasive adenocarcinoma on the basis of tissue pathology. By HRCT assessment, 57 patients (29\%) had lesions that classified them in the HL group; the remaining 138 patients had lesions that classified them into the TL group. There were no significant differences in with respect to demographic characteristics and tissue pathology between the two groups.

Table 2 includes the results of an evaluation based on both HRCT and 3D CT. There were no patients with lesions diagnosed as cTis (clinical T in situ) in the HL group. The solid tumor size measured by HRCT was generally larger in the HL group compared to the TL group $(\mathrm{P}=0.045)$. There were no statistical differences between HL and TL groups for the evaluation of whole tumor volume and the solid tumor volume by 3D CT. Inter-observer agreement with respect to measurements for solid tumor size on HRCT were lower in the HL group (ICC $=0.561$ ) than in the TL group $(\mathrm{ICC}=0.965)$ (Table 3).
Figure 3 shows ROC curve that predicted invasive adenocarcinoma based on the solid tumor volume. The AUC and the optimal cut-off value were 0.881 and $225 \mathrm{~mm}^{3}$, respectively.

We further assessed accuracy, sensitivity, and specificity for the prediction of invasive adenocarcinoma based on solid tumor size as determined by HRCT and solid tumor volume as determined by 3D CT (Table 4). We set the cutoff value for solid tumor volume at $225 \mathrm{~mm}^{3}$ as per the ROC curve. Among all the patients considered together, the accuracy, sensitivity and specificity based on the solid tumor volume were similar to parameters based on solid tumor size. However, among those in the HL group, the specificity based on the solid tumor volume $(65.5 \%)$ was higher than that based on the solid tumor size $(44.8 \%)$; the difference between the two parameters approached statistical significance $(\mathrm{P}=0.070)$.

\section{Discussion}

In the present study, we evaluated the predictive value for pathological invasiveness of clinical stage IA adenocarcinoma in a comparison of two imaging modalities, specifically using measures of solid tumor size from HRCT and solid tumor volume by 3D CT. Preoperative estimates of tumor size provided by imaging modalities are crucial for determining appropriate treatment strategy. Current protocols indicate that HRCT should be performed prior to any treatment in order to evaluate the size of small 
Table 1 Patient characteristics

\begin{tabular}{|c|c|c|c|c|}
\hline Variables & Total $(\mathrm{n}=195), \mathrm{n}[\%]$ & HL group (n=57), n [\%] & TL group ( $n=138), n[\%]$ & $P$ value \\
\hline \multicolumn{5}{|l|}{ Sex } \\
\hline Male & 84 [43] & 21 [37] & $63[46]$ & \multirow[t]{2}{*}{0.258} \\
\hline Female & $111[57]$ & 36 [63] & $75[54]$ & \\
\hline Yes & $22[11]$ & 7 [12] & 15 [11] & \multirow[t]{2}{*}{0.784} \\
\hline No & $173[89]$ & $50[88]$ & $123[89]$ & \\
\hline \multicolumn{5}{|l|}{ Surgical procedure } \\
\hline Lobectomy & $129[66]$ & $40[70]$ & 89 [64] & 0.446 \\
\hline AIS/MIA & $86[44]$ & $29[51]$ & $57[41]$ & \multirow[t]{2}{*}{0.221} \\
\hline Invasive Ad & $109[56]$ & 28 [49] & $81[59]$ & \\
\hline \multicolumn{5}{|l|}{ Lymphatic invasion } \\
\hline Positive & $21[11]$ & $4[7]$ & 17 [12] & \multirow[t]{2}{*}{0.277} \\
\hline Negative & $174[89]$ & 53 [93] & $121[88]$ & \\
\hline \multicolumn{5}{|l|}{ Vascular invasion } \\
\hline Positive & 27 [14] & 7 [12] & $20[14]$ & \multirow[t]{2}{*}{0.684} \\
\hline Negative & $168[86]$ & $50[88]$ & $118[86]$ & \\
\hline
\end{tabular}

AIS, adenocarcinoma in situ; MIA, minimally invasive adenocarcinoma; Ad, adenocarcinoma; HL, Hazy lesion; TL, typical lesion

adenocarcinomas. However, we find that some lesions are difficult to measure on HRCT because of irregular shape and unclear boundaries between the solid and non-solid regions of the tumor. To address this problem, we examined these tumors with $3 \mathrm{D}$ CT imaging technology; this modality provides quantitative assessments of whole and solid tumor volume.

We failed to show superiority of 3D CT imaging over existing HRCT technology for predicting invasive lung adenocarcinoma. However, in the HL group, the solid tumor volume by 3D CT tended to be more specific and a better predictor of an invasive lesion than that provided by solid tumor size as determined by HRCT. Toward this end, we should consider the potential bias when measuring the solid tumor size on HRCT. Among these biases, the observers tasked with scoring these lesions might be biased psychologically toward larger sizes so as to avoid the risk of underestimation of tumor invasiveness, and thus provide the patient with inappropriate therapy, such as limited resection. In other words, preoperative evaluation may result in an overestimate of tumor size so that in the end one is ensuring curability; this is especially likely in HL group, in which determining tumor size is more difficult. By contrast, 3D CT imaging provides a semi-automatic evaluation of the solid tumor volume; as such, the psychological bias will be reduced if not eliminated.

About the sensitivity, we could not show the superiority of the solid tumor volume in HL group. We cannot provide a clear explanation, but the reason for this result might be that 3D CT categorizes solids and non-solid regions using 
Table 2 Evaluation of the tumors using HRCT and 3D CT in each group

\begin{tabular}{|c|c|c|c|c|}
\hline Variables & Total $(n=195)$ & HL group $(n=57)$ & TL group $(n=138)$ & $P$ value \\
\hline Tis & 19 [10] & $0[0]$ & 19 [14] & \\
\hline T1mi & $40[21]$ & 16 [28] & 24 [17] & \\
\hline T1a-T1c & $136[69]$ & $41[72]$ & 95 [69] & \\
\hline Whole tumor size (cm) & & & & 0.579 \\
\hline Range & $0.5-4.4$ & $0.6-4.1$ & $0.5-3.9$ & \\
\hline Mean \pm SD & $1.93 \pm 0.76$ & $1.97 \pm 0.74$ & $1.92 \pm 0.76$ & \\
\hline Solid tumor size $(\mathrm{cm})$ & & & & 0.045 \\
\hline \multicolumn{5}{|l|}{ 3D CT findings } \\
\hline Whole tumor volume $\left(\mathrm{mm}^{3}\right)$ & & & & 0.466 \\
\hline Range & $102.0-22,876.5$ & $214.1-1,8981.2$ & $102.0-2,2876.5$ & \\
\hline Mean \pm SD & $2,975.0 \pm 3,426.7$ & $2,994.8 \pm 3,450.0$ & $2,966.8 \pm 3,426.7$ & \\
\hline Solid tumor volume $\left(\mathrm{mm}^{3}\right)$ & & & & 0.329 \\
\hline Range & $0-7,044$ & $0.5-4,839.1$ & $0-7,044$ & \\
\hline Mean \pm SD & $1,026.0 \pm 1,364.5$ & $779.3 \pm 1,358.0$ & $1,127.9 \pm 1,361.3$ & \\
\hline
\end{tabular}

HRCT, High-resolution computed tomography; 3D CT, three-dimensional computed tomography; SD, standard deviation; HL, Hazy lesion; TL, typical lesion.

Table 3 Analysis of inter-observer agreements for measurement of solid tumor size

\begin{tabular}{lc}
\hline Group & ICC $(95 \% \mathrm{Cl})$ \\
\hline HL group & $0.561(0.283-0.737)$ \\
TL group & $0.965(0.951-0.975)$ \\
\hline
\end{tabular}

ICC, intra-class correlation coefficient; $\mathrm{Cl}$, confidence interval; $\mathrm{HL}$, hazy lesion; TL, typical lesion.

$-300 \mathrm{HU}$ as a cutoff value uniformly.

There are some relatively recent reports that discuss the utility of 3D CT imaging for prediction of the pathologic features and outcome in patients diagnosed with non-small cell lung cancer (NSCLC) (19-22). Eriguchi et al. explored the utility of quantitative CT histograms calculated by Synapse Vincent together with maximum standardized uptake values (SUVmax) on F-18 fluorodeoxyglucosepositron emission tomography CT (20). They reported that the combination of these modalities provided clear and accurate predictions histologic subtypes in patients with stage I adenocarcinoma. Likewise, Furumoto et al. reported that solid tumor volume measured by $3 \mathrm{D}$ CT was an independent predictor of disease-free survival; integration of the solid tumor volume and SUVmax was the most powerful predictor (19). However, in these studies, the authors made no distinction between lung nodules that were relatively easy $v s$. those that were difficult to evaluate on HRCT; the latter were the focus of the work presented here. To the best of our knowledge, this is the first study that compared the efficacy of 3D CT $v s$. HRCT for predicting the invasiveness of lung adenocarcinoma with a particular focus on lesions that are difficult to evaluate by HRCT.

There are some limitations to the present study. First, the present study is retrospective in nature with a small number of cases from single institute. The study includes several potential biases and does not have enough statistical power to permit us to draw substantial conclusions. A largescale study to validate these findings is needed. Another 
limitation relates to the perceived universality of the $3 \mathrm{D}$ assessment provided vis Synapse Vincent software. Via the algorithms currently included with this program, whole and solid tumor volume of a tumor are calculated automatically. However, in a previous iteration, we needed to align both ends of the tumor in a straight line in an axial plane; this is similar to the methodology used for assessment on HRCT, and introduces the possibility of error and discrepancies between software packages. On this last point, Nemec

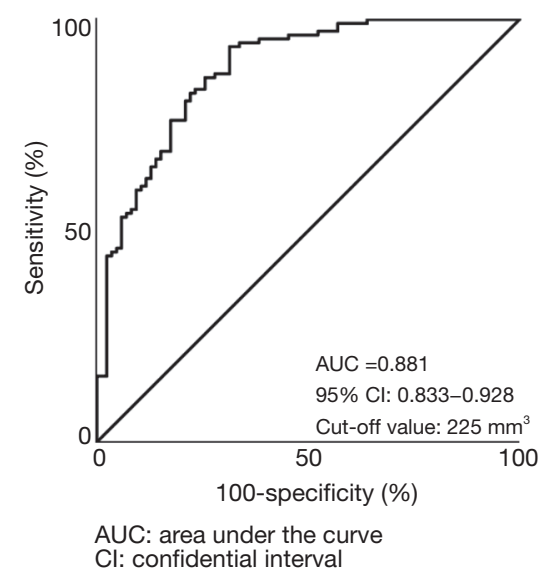

Figure 3 The area under the receiver operating characteristic curve for tumor invasiveness determined using the solid volume as determined by 3D CT. et al. reported only small differences when analyzing lung volumes using different software packages (23). However, we acknowledge that the cut-off values for solid tumor volume were set to an arbitrary value based on data from the ROC curve and not universally available parameters. By contrast, for our measurements of the solid tumor size, we defined the cut-off value as $5 \mathrm{~mm}$ which is the universal value as per the TNM classification. We did not evaluate the reproducibility of 3D CT analysis. However, interobserver and intraobserver errors will remain minimal because we just need to do the simple task, tracing both ends of the tumor in a straight line in axial plane for 3DCT analysis. Finally, the decision criteria for distinguishing between the two groups, whether measuring the solid tumor size is difficult or easy, is by definition subjective and ambiguous. However, we note that inter-observer agreement of cases assigned to the HL group was clearly lower than that of cases assigned to the TL group, an observation that confirms our contention that solid tumor sizes in the former group are indeed difficult to measure accurately. Despite these limitations, the present study provides new insights into 3D CT imaging as a clinical tool that all types of physicians might employ.

\section{Conclusions}

In summary, 3D CT imaging was equivalent to HRCT

Table 4 Predictive power for invasive adenocarcinoma by solid tumor size $v s$. volume

\begin{tabular}{|c|c|c|c|}
\hline Group & Solid tumor size (cut-off: $5 \mathrm{~mm}$ ) & Solid tumor volume (cut-off: $225 \mathrm{~mm}^{3}$ ) & $P$ value \\
\hline \multicolumn{4}{|l|}{ Total $(n=195)$} \\
\hline Accuracy (\%) & 81.5 & 82.6 & 0.845 \\
\hline Sensitivity (\%) & 95.4 & 93.6 & 0.727 \\
\hline Specificity (\%) & 64.0 & 68.6 & 0.481 \\
\hline \multicolumn{4}{|l|}{$\mathrm{HL}$ group $(\mathrm{n}=57)$} \\
\hline Accuracy (\%) & 82.6 & 84.1 & 0.109 \\
\hline Sensitivity (\%) & 92.9 & 92.9 & 1.000 \\
\hline Specificity (\%) & 44.8 & 65.5 & 0.070 \\
\hline \multicolumn{4}{|l|}{ TL group $(n=138)$} \\
\hline Accuracy (\%) & 77.6 & 67.2 & 0.454 \\
\hline Sensitivity (\%) & 96.3 & 93.8 & 0.687 \\
\hline Specificity (\%) & 73.7 & 70.2 & 0.754 \\
\hline
\end{tabular}

$\mathrm{HL}$, hazy lesion; TL, typical lesion. 
with respect to the efficacy of predicting invasive adenocarcinoma. However, 3D CT imaging might be more effective than HRCT for lesions which have irregular shape and are difficult to evaluate using the latter modality. 3D CT imaging has great potential toward generating more precise and accurate surgical strategies.

\section{Acknowledgments}

The authors would like to thank Enago (www.enago.jp) for the English language review.

Funding: This research was funded by the Cancer Institute Hospital of Japanese Foundation for Cancer Research.

\section{Footnote}

Reporting Checklist: The authors have completed the STROBE reporting checklist. Available at http://dx.doi. org/10.21037/jtd-20-2131

Peer Review File: Available at http://dx.doi.org/10.21037/jtd20-2131

Data Sharing Statement: Available at http://dx.doi. org/10.21037/jtd-20-2131

Conflicts of Interest: All authors have completed the ICMJE uniform disclosure form (available at http://dx.doi. org/10.21037/jtd-20-2131). The authors have no conflicts of interest to declare.

Ethical Statement: The authors are accountable for all aspects of the work in ensuring that questions related to the accuracy or integrity of any part of the work are appropriately investigated and resolved. The study was conducted in accordance with the Declaration of Helsinki (as revised in 2013). Data collection and analysis were approved by the Institutional Review Board of the Cancer Institute Hospital of Japanese Foundation for Cancer Research (2019-1235). The need to obtain written informed consent from each patient was waived because of the retrospective nature of the study and anonymity of the subjects.

Open Access Statement: This is an Open Access article distributed in accordance with the Creative Commons Attribution-NonCommercial-NoDerivs 4.0 International License (CC BY-NC-ND 4.0), which permits the noncommercial replication and distribution of the article with the strict proviso that no changes or edits are made and the original work is properly cited (including links to both the formal publication through the relevant DOI and the license). See: https://creativecommons.org/licenses/by-nc-nd/4.0/.

\section{References}

1. Matsuguma H, Yokoi K, Anraku M, et al. Proportion of ground-glass opacity on high-resolution computed tomography in clinical T1 N0 M0 adenocarcinoma of the lung: a predictor of lymph node metastasis. J Thorac Cardiovasc Surg 2002;124:278-84.

2. Okada M, Nishio W, Sakamoto T, Uchino K, Tsubota N. Discrepancy of computed tomographic image between lung and mediastinal windows as a prognostic implication in small lung adenocarcinoma. Ann Thorac Surg 2003;76:1828-32.

3. Takamochi K, Nagai K, Yoshida J, et al. The role of computed tomographic scanning in diagnosing mediastinal node involvement in non-small cell lung cancer. J Thorac Cardiovasc Surg 2000;119:1135-40.

4. Tsutani Y, Miyata Y, Nakayama H, et al. Prognostic significance of using solid versus whole tumor size on High-resolution computed tomography for predicting pathologic malignant grade of tumors in clinical stage IA lung adenocarcinoma: a multicenter study. J Thorac Cardiovasc Surg 2012;143:607-12.

5. Uehara H, Tsutani Y, Okumura S, et al. Prognostic role of positron emission tomography and highresolution computed tomography in clinical stage IA lung adenocarcinoma. Ann Thorac Surg 2013;96:1958-65.

6. Hattori A, Matsunaga T, Takamochi K, et al. Surgical resection for clinical-Stage I radiological pure-solid lung cancer that met the current high risk criteria. Jpn J Clin Oncol 2017;47:630-8.

7. Hattori A, Takamochi K, Matsunaga T, et al. Oncological outcomes of sublobar resection for clinical-stage IA high-risk non-small cell lung cancer patients with a radiologically solid appearance on computed tomography. Gen Thorac Cardiovasc Surg 2016;64:18-24.

8. Goldstraw P, Chansky K, Crowley J, et al. The IASLC lung Cancer Staging project: proposals for revision of the TNM stage groupings in the forthcoming (Eighth) edition of the TNM classification for lung Cancer. J Thorac Oncol 2016;11:39-51.

9. Son JY, Lee HY, Lee KS, et al. Quantitative CT analysis of pulmonary ground-glass opacity nodules for the distinction of invasive adenocarcinoma from pre-invasive or minimally 
invasive adenocarcinoma. PLoS One 2014;9:e104066.

10. Lee HY, Lee KS. Ground-glass opacity nodules: histopathology, imaging evaluation and clinical implications. J Thorac Imaging 2011;26:106-18.

11. Travis WD, Garg K, Franklin WA, et al. Evolving concepts in the pathology and computed tomography imaging of lung adenocarcinoma and bronchioloalveolar carcinoma. J Clin Oncol 2005;23:3279-87.

12. Ikeda K, Awai K, Mori T, et al. Differential diagnosis of ground-glass opacity nodules: CT number analysis by three-dimensional computerized quantification. Chest 2007;132:984-90.

13. Hagiwara M, Shimada Y, Kato Y, et al. High-quality 3-dimensional image simulation for pulmonary lobectomy and segmentectomy: results of preoperative assessment of pulmonary vessels and short-term surgical outcomes in consecutive patients undergoing video-assisted thoracic surgerydagger. Eur J Cardiothorac Surg 2014;46:e120-6.

14. Ikeda N, Yoshimura A, Hagiwara M, et al. Three dimensional computed tomography lung modeling is useful in simulation and navigation of lung cancer surgery. Ann Thorac Cardiovasc Surg 2013;19:1-5.

15. Oizumi H, Kanauchi N, Kato H, et al. Anatomic thoracoscopic pulmonary segmentectomy under 3-dimensional multidetector computed tomography simulation: a report of 52 consecutive cases. J Thorac Cardiovasc Surg 2011;141:678-82.

16. Solomon B, Bizekis C, Dellis SL, et al. Simulating videoassisted thoracoscopic lobectomy: a virtual reality cognitive

Cite this article as: Kawaguchi $\mathrm{Y}$, Nakao M, Omura K, Iwamoto N, Ozawa H, Kondo Y, Ichinose J, Matsuura Y, Okumura S, Mun M. The utility of three-dimensional computed tomography for prediction of tumor invasiveness in clinical stage IA lung adenocarcinoma. J Thorac Dis 2020;12(12):7218-7226. doi: 10.21037/jtd-20-2131 task simulation. J Thorac Cardiovasc Surg 2011;141:249-55.

17. Kanzaki M, Maeda H, Wachi N, et al. Complete videoassisted thoracoscopic multi-subsegmentectomy based on patients' specific virtual 3-D pulmonary models. Asian J Endosc Surg 2013;6:110-5.

18. Saji H, Inoue $T$, Kato $Y$, et al. Virtual segmentectomy based on high-quality three-dimensional lung modelling from computed tomography images. Interact Cardiovasc Thorac Surg 2013;17:227-32.

19. Furumoto H, Shimada Y, Imai K, et al. Prognostic impact of the integration of volumetric quantification of the solid part of the tumor on 3DCT and FDG-PET imaging in clinical stage IA adenocarcinoma of the lung. Lung Cancer 2018;121:91-6.

20. Eriguchi D, Shimada Y, Imai K, et al. Predictive accuracy of lepidic growth subtypes in early-stage adenocarcinoma of the lung by quantitative CT histogram and FDG-PET. Lung Cancer 2018;125:14-21.

21. Takenaka T, Yamazaki K, Miura N, et al. The prognostic impact of tumor volume in patients with clinical stage IA non-small cell lung cancer. J Thorac Oncol 2016;11:1074-80.

22. Shimada Y, Furumoto H, Imai K, et al. Prognostic value of tumor solid-part size and solid-part volume in patients with clinical stage I non-small cell lung cancer. J Thorac Dis 2018; 10:6491-500.

23. Nemec SF, Molinari F, Dufresne V, et al. Comparison of four software of four software packages for CT lung volumetry in healthy individuals. Eur Radiol 2015;25:1588-97. 